\title{
ON REDUCIBILITY OF SOME OPERATOR SEMIGROUPS AND ALGEBRAS ON LOCALLY CONVEX SPACES
}

\author{
EDVARD KRAMAR
}

Received 9 March 2005 and in revised form 20 October 2005

A generalization of some results from normed spaces, concerning reducibility and triangularizability of semigroups and algebras of operators, to locally convex spaces is given.

\section{Introduction}

Let $X$ be a complex Hausdorff locally convex space. A system of seminorms $P$ inducing the topology on $X$ will be called a calibration. We denote by $\mathscr{P}(X)$ the collection of all calibrations on $X$. For a given $P \in \mathscr{P}(X)$ let $P=\left\{p_{\alpha}: \alpha \in \Delta\right\}$, where $\Delta$ is some index set and for each $\alpha \in \Delta$ denote $U_{\alpha}=\left\{x: p_{\alpha}(x) \leq 1\right\}$. Let us denote by $\mathscr{L}(X)$ the set of all linear continuous operators on $X$, by $\mathscr{K}(X)$ the set of compact operators on $X(T \in$ $\mathscr{K}(X)$ if there exists a neighbourhood $U_{\gamma}$ such that $T\left(U_{\gamma}\right)$ is a relatively compact set), by $\mathscr{F}(X)$ the set of all finite-rank operators and by $\mathscr{L} B(X)$ the set of all locally bounded operators (there is some neighbourhood $U_{\gamma}$ such that $T\left(U_{\gamma}\right)$ is bounded). The topology of bounded convergence on $\mathscr{L}(X)$ and on $X^{\prime}$ will be denoted by $\tau_{b}$. By $X_{b}^{\prime}$ we will denote the topological space $\left(X^{\prime}, \tau_{b}\right)$. We will denote by $\mathscr{R}(T)$ the range of $T$ and by $\mathcal{N}(T)$ the null space of $T$. For a given $T \in \mathscr{L}(X)$ the number $\lambda \in \mathbb{C}$ is in the resolvent set of $T$ if and only if $(\lambda I-T)^{-1}$ exists in $\mathscr{L}(X)$. The spectrum $\sigma(T)$ is the complement of the resolvent set and by $r(T)=\sup \{|\lambda|: \lambda \in \sigma(T)\}$ we denote the spectral radius of $T$. An operator $T$ is quasinilpotent if $\sigma(T)=\{0\}$. A closed subspace $M$ in $X$ is an invariant subspace of an operator $T$ if $T(M) \subseteq M$. A collection of linear operators is reducible if it has a common nontrivial invariant subspace and is irreducible otherwise. If a family $\mathscr{A} \subset \mathscr{L}(X)$ is an algebra, it is irreducible if and only if it is transitive, that is, the set $\mathscr{A} x:=\{T x: T \in \mathscr{A}\}$ is dense in $X$ for each $x \neq 0$. For $P \in \mathscr{P}(X)$ and $p_{\alpha} \in P$ let $J_{\alpha}$ denote the null space of $p_{\alpha}$ and $X_{\alpha}$ the quotient space $X / J_{\alpha}$. It is a normed space with the norm $\left\|x_{\alpha}\right\|_{\alpha}:=p_{\alpha}(x)$, where $x_{\alpha}=x+J_{\alpha}$. Let $T \in \mathscr{L}(X)$ be such that $T\left(J_{\alpha}\right) \subset J_{\alpha}$ then the corresponding operator $T_{\alpha}$ on $X_{\alpha}$ is well defined by $T_{\alpha}\left(x_{\alpha}\right)=T x+J_{\alpha}$.

\section{The results}

Lemma 2.1. Let $X$ be a locally convex space and $A$ a transitive $\tau_{b}$-closed algebra of continuous operators on $X$ which contains a nonzero finite-rank operator. Then there exists a 
$\tau_{b}$-closed subspace $\Phi$ in $X_{b}^{\prime}$ such that

(i) A contains all rank-one operators of the form $x \otimes f, x \in X, f \in \Phi$,

(ii) if $f(z)=0$ for each $f \in \Phi$, then $z=0$.

Proof. Let $F \in \mathscr{A}$ be a nonzero finite-rank operator. Denote $E_{1}=\mathscr{R}(F)$ and $\mathscr{A}_{1}:=F \mathscr{A}$. It is clear that each $T \in \mathscr{A}_{1}$ maps $E_{1}$ to $E_{1}$ and the restriction $\left.\mathscr{A}_{1}\right|_{E_{1}}$ is a transitive algebra of operators on a finite-dimensional space. By Burnside's theorem [11] it follows that $\left.\mathscr{A}_{1}\right|_{E_{1}}=\mathscr{L}\left(E_{1}\right)$. Especially, there is some $A_{0} \in \mathscr{A}$ such that $\left.F A_{0}\right|_{E_{1}}$ has rank one, hence $F A_{0} F$ is a rank-one operator in $\mathscr{A}$ of the form $x_{0} \otimes f_{0}, x_{0} \in X$, and $f_{0} \in X^{\prime}$. Choose arbitrary nonzero $x \in X$. Since $\mathscr{A} x_{0}$ is dense in $X$, there is some net of operators $\left\{A_{\delta}\right\} \subset \mathscr{A}$ such that $A_{\delta} x_{0} \rightarrow x$. For any chosen seminorm $q_{\alpha}^{M}$ defining the topology $\tau_{b}$ we have

$$
q_{\alpha}^{M}\left(\left(A_{\delta} x_{0}\right) \otimes f_{0}-x \otimes f_{0}\right)=\sup _{y \in M}\left|f_{0}(y)\right| p_{\alpha}\left(A_{\delta} x_{0}-x\right) \leq c \cdot p_{\alpha}\left(A_{\delta} x_{0}-x\right) .
$$

Since the right-hand side tends to zero, the same holds for the left-hand side, thus $x \otimes f_{0} \in$ $\mathcal{A}$. Let us define $\Phi:=\left\{f \in X^{\prime}: x \otimes f \in \mathcal{A}\right.$, for all $\left.x \in X\right\}$. Choose $\left\{f_{\delta}\right\} \subset \Phi$ a net which is $\tau_{b}$-convergent to some $f$, and $x \in X$ arbitrary, then for any seminorm $q_{\alpha}^{M}$ we have

$$
q_{\alpha}^{M}\left(x \otimes f_{\delta}-x \otimes f\right)=\sup _{y \in M}\left|\left(f_{\delta}-f\right)(y)\right| p_{\alpha}(x) .
$$

Since $\mathscr{A}$ is $\tau_{b}$-closed we have $x \otimes f \in \mathscr{A}$, hence $f \in \Phi$. Thus, $\Phi$ is a nontrivial $\tau_{b}$-closed subspace satisfying (i). To verify (ii), let $f(z)=0$ for each $f \in \Phi$. Choose a nonzero $f_{0} \in$ $\Phi$ and for each $A \in \mathscr{A}$ define $f_{1}:=A^{\prime} f_{0}$, where $A^{\prime}$ is the adjoint operator of $A$. Since $x \otimes f_{1}=x \otimes A^{\prime} f_{0}=\left(x \otimes f_{0}\right) A \in A$, for any $x \in X$, we have $f_{1} \in \Phi$, hence $f_{1}(z)=0$, that is $f_{0}(A z)=0$ for each $A \in \mathscr{A}$. If there were $z \neq 0$, then $f_{0}$ would be zero on a dense set $\mathscr{A} z$ and consequently identically zero, which is a contradiction.

Corollary 2.2. Let $X$ be a semireflexive locally convex space and $A$ a transitive $\tau_{b}$-closed algebra of continuous operators which contains a nonzero finite-rank operator. Then $A$ contains all finite-rank operators.

Proof. It is sufficient to show that $\mathscr{A}$ contains all rank-one operators and this will be in case $\Phi=X^{\prime}$. If there were $\Phi \neq X^{\prime}$, then by the Hahn-Banach theorem there would be some nonzero $F \in\left(X_{b}^{\prime}\right)^{\prime}$, such that $\left.F\right|_{\Phi}=0$. Since $X$ is semireflexive, there is some nonzero $y \in X$ such that $F(f)=f(y)$ for all $f \in X^{\prime}$ and then $f(y)=F(f)=0$ for all $f \in \Phi$. By (ii) in the previous lemma then $y=0$, which is a contradiction.

A linear operator $T$ is called nuclear if it can be written in the form

$$
T x=\sum_{j=1}^{\infty} \lambda_{j} c_{j}(x) a_{j}, \quad x \in X,
$$

where $\left\{c_{j}\right\}$ is an equicontinuous sequence in $X^{\prime},\left\{\lambda_{j}\right\} \in l_{1}$, and $\left\{a_{j}\right\}$ is a sequence contained in an absolutely convex bounded set $B$ in $X$, such that $X_{B}:=\bigcup\{n B: n \in \mathbb{N}\}$ is a complete normed subspace in $X$ with respect to the Minkowski's functional of the set $B$. (see, e.g., [8]). It is easy to see that the family of nuclear operators is an ideal in $\mathscr{L}(X)$ and that each nuclear operator is also compact. 
Corollary 2.3. Let $X$ be a semireflexive locally convex space and $A$ a transitive algebra of continuous operators such that $\overline{\left(\mathscr{A}, \tau_{b}\right)}$ contains a nonzero finite-rank operator. Then $\overline{\left(\mathscr{A}, \tau_{b}\right)}$ contains all nuclear operators.

Proof. By Corollary 2.2, $\overline{\left(\mathscr{A}, \tau_{b}\right)} \supset \mathscr{F}(X)$, since each nuclear operator can be $\tau_{b}$-approximated by finite-rank operators by [1], the conclusion follows.

For a given set $\mathcal{M}$ of compact operators let us denote by $\widetilde{M}$ the set of all $A \in \mathscr{K}(X)$ which are $\tau_{b}$-limits of some sequence $\left\{A_{n}\right\} \subset \mathcal{M}$. By [5, Proposition 1] it follows that if $X$ is a barreled locally convex space and $\mathscr{S}$ a semigroup of compact operators on $X$, then $\tilde{\mathscr{S}}$ is a semigroup too.

Proposition 2.4. Let $X$ be a barreled locally convex space and $\mathscr{S}$ a semigroup of compact operators. If $A \in \mathscr{S}$ is such that $r(A) \neq 0$ then there exists a sequence $\left\{n_{i}\right\}$ of integers such that one of the following assertions holds:

(a) $A^{n_{i}} \stackrel{\tau_{b}}{\rightarrow} E$, where $E$ is idempotent, or

(b) $\alpha_{i} A^{n_{i}} \stackrel{\tau_{b}}{\rightarrow} E$, for some scalar sequence $\left\{\alpha_{i}\right\}$, where $E$ is nilpotent.

In both cases $E \in \widetilde{\mathbb{R}^{+} \mathscr{S}}$ and is of finite rank.

Proof. Following the first part of the proof of [6, Proposition 3] we can find a sequence of operators from $\mathscr{S}$ with the above property.

Theorem 2.5. Let $X$ be a barreled locally convex space and $A$ a $\tau_{b}$-closed transitive algebra in $\mathscr{L}(X)$ which contains a nonzero compact operator. Then $\mathscr{A} \cap \mathscr{F}(X)$ is a nontrivial transitive algebra.

Proof. Define $\mathscr{C}=\mathscr{A} \cap \mathscr{K}(X)$, then it is an ideal in $\mathscr{A}$ and by [6, Lemma 5] it is transitive too. Let $K$ be a nonzero operator in $\mathscr{C}$. Then by Lomonosov's theorem for locally convex spaces [3] there is some $A \in \mathscr{C}$ such that $r(A K) \neq 0$. By Proposition 2.4, $\widetilde{\mathscr{C}} \cap \mathscr{F}(X) \neq\{0\}$. Since $\mathscr{A}$ is $\tau_{b}$-closed, it is easy to see that $\widetilde{\mathscr{C}}=\mathscr{C}$. Clearly, $\mathscr{A} \cap \mathscr{F}(X)=\mathscr{C} \cap \mathscr{F}(X)$ is an ideal in $\mathscr{A}$ and it is transitive too.

Corollary 2.6. Let $X$ be a barreled semireflexive locally convex space and $A$ a $\tau_{b}$-closed transitive algebra in $\mathscr{L}(X)$ which contains a nonzero compact operator. Then $\mathcal{A}$ contains all finite-rank operators.

Proof. By Theorem 2.5 and Corollary 2.2, the conclusion follows.

Corollary 2.7. Let $X$ be a barreled semireflexive locally convex space and $A$ a transitive $\tau_{b}$-closed algebra in $\mathscr{L}(X)$ which contains a nonzero compact operator. If $X$ has the property that each compact operator on $X$ is $\tau_{b}$-limit of a net of finite-rank operators, then $A$ contains all compact operators.

Proposition 2.8. Let $X$ be a semireflexive locally convex space, $\mathscr{S}$ a semigroup in $\mathscr{F}(X)$, and $\phi$ a nontrivial $\tau_{b}$-continuous linear functional on $\mathscr{F}(X)$. If $\phi$ is identically zero on $\mathscr{Y}$, then $\mathscr{S}$ is reducible.

Proof. Let us suppose that $\mathscr{S}$ is irreducible. Then the algebra $\mathscr{A}$ generated by $\mathscr{S}$ is also irreducible and the same holds for $\overline{\left(\mathscr{A}, \tau_{b}\right)}$. By Corollary 2.2, $\overline{\left(\mathscr{A}, \tau_{b}\right)} \supset \mathscr{F}(X)$. Since $\phi$ is equal to zero on $\mathscr{Y}$, it is equal to zero also on $\mathscr{F}(X)$, which is a contradiction. 
With respect to the strong topology $\tau_{s}$ on $\mathscr{L}(X)$ the following theorem is proven in [8]. TheORem 2.9. Let $X$ be a locally convex space and $\mathscr{A}$ a transitive algebra in $\mathscr{L}(X)$, such that $\overline{\left(\mathscr{A}, \tau_{s}\right)}$ contains a nontrivial compact operator. Then $\mathscr{A}$ is $\tau_{s}$-dense in $\mathscr{L}(X)$.

Corollary 2.10. Let $X$ be a locally convex space and A a nonscalar operator commuting with a nonzero compact operator. Then A has a nontrivial hyperinvariant subspace.

Proof. Denote by $\mathscr{A}:=(A)^{\prime}$ the commutant of $A$. Clearly, it is an algebra and let us prove that it is $\tau_{s}$-closed. Choose any net $\left\{B_{\delta}\right\}$ in $\mathscr{A}$ which is strongly convergent to some $B$. Then for any seminorm $q_{x}^{\alpha}$ for the strong topology one has

$$
\begin{aligned}
q_{x}^{\alpha}(B A-A B) & =p_{\alpha}((B A-A B) x) \leq p_{\alpha}\left(\left(B-B_{\delta}\right) A x\right)+p_{\alpha}\left(A\left(B-B_{\delta}\right) x\right) \\
& \leq q_{y}^{\alpha}\left(B-B_{\delta}\right)+c_{\alpha} q_{x}^{\beta}\left(B-B_{\delta}\right),
\end{aligned}
$$

where $y=A x$. Since the right-hand side is arbitrary small, the left-hand side is zero. Since $q_{x}^{\alpha}$ is arbitrary, we have $B A-A B=0$. If $\mathscr{A}$ were transitive, then by the above theorem, it would be equal to $\mathscr{L}(X)$ and consequently $A=\lambda I$ for some complex number $\lambda$, which is a contradiction.

Corollary 2.11. Let $X$ be a locally convex space and $A, B \in \mathscr{L}(X)$ two commuting operators, where $B$ is nonscalar and commutes with a nonzero compact operator. Then $A$ has a nontrivial invariant subspace.

Proof. By Corollary 2.10, $B$ has a hyperinvariant subspace which is invariant for $A$.

Corollary 2.12. Let $X$ be a locally convex space and $\mathscr{S}$ a semigroup of operators such that $\overline{\left(\mathscr{Y}, \tau_{s}\right)}$ contains a nonzero compact operator and $\phi$ a nontrivial $\tau_{s}$-continuous linear functional on $\mathscr{L}(X)$ such that $\left.\phi\right|_{\mathscr{S}}=0$. Then $\mathscr{S}$ is reducible.

Proof. Let $\mathscr{S}$ be irreducible, then the algebra $\mathscr{A}$ generated by $\mathscr{S}$ is also irreducible and then by Theorem 2.9 , it is strongly dense in $\mathscr{L}(X)$. Clearly, $\phi$ is equal to zero also on $\mathscr{A}$ and thus on $\mathscr{L}(X)$, which is a contradiction.

Corollary 2.13. Let $X$ be a locally convex space and $\mathscr{E}$ a commutative family of compact operators on $X$. Then $\mathscr{E}$ is reducible.

Proof. Choose a nonzero $A \in \mathscr{E}$, by Corollary 2.10 it has a nontrivial hyperinvariant subspace which is then an invariant subspace for $\mathscr{E}$.

Let $\phi$ be a functional on a semigroup $\mathscr{S} \subset \mathscr{L}(X)$. By [11] $\phi$ is permutable on a family $\mathscr{E} \subset \mathscr{S}$ if for any $A_{1}, A_{2}, \ldots, A_{n} \in \mathscr{E}$ and any permutation $\tau$ of $\{1,2, \ldots, n\}$ we have $\phi\left(A_{1}, A_{2}, \ldots, A_{n}\right)=\phi\left(A_{\tau(1)}, A_{\tau(2)}, \ldots, A_{\tau(n)}\right)$.

Proposition 2.14. Let $X$ be a semireflexive locally convex space and $\phi$ a nontrivial $\tau_{b}$ continuous linear functional on $\mathscr{F}(X)$. Let $\mathscr{E}$ be a family of finite-rank operators such that $\phi$ is permutable on $\mathscr{E}$. Then $\mathscr{E}$ is reducible.

Proof. Since $\phi$ is permutable on $\mathscr{E}$, it is also permutable on the algebra $\mathscr{A}$ generated by $\mathscr{E}$. In view of Corollary 2.13 we can assume that $\mathscr{E}$ is noncommutative. Then there are 
$A, B \in \mathscr{E}$ such that $C:=A B-B A \neq 0$. Denote by $\mathscr{F}$ the ideal in $\mathscr{A}$ generated by $C$. Clearly, $\phi(S C T)=0$ for each $S, T \in \mathscr{A}$, consequently, $\left.\phi\right|_{\mathscr{g}}=0$. Hence, by Proposition 2.8, $\mathscr{E}$ is reducible, then by [6], $\mathscr{A}$ is reducible and $\mathscr{E}$ is reducible too.

Corollary 2.15. Let $X$ be a semireflexive locally convex space, $\mathscr{Y}$ a semigroup of finite-rank operators, and $\phi$ a nontrivial $\tau_{b}$-continuous linear functional on $\mathscr{F}(X)$. Then $\mathscr{S}$ is reducible if one of the following conditions holds:

(i) $\phi$ is multiplicative on $\mathscr{Y}$,

(ii) $\phi$ is constant on $\mathscr{Y}$.

For fixed nonzero $x_{0} \in X, f \in X^{\prime}$ and a subspace $\mathcal{M}$ in $\mathscr{L}(X)$ let us define (as in [11]) the so-called coordinate functional by the relation $\phi(T):=f\left(T x_{0}\right), T \in \mathcal{M}$. For this class of functionals we do not need semireflexivity of the space.

Lemma 2.16. Let $X$ be a locally convex space, then the coordinate functional $\phi$ is $\tau_{b}$-continuous on $\mathscr{L}(X)$ if and only if $f$ is continuous on $X$.

Proof. If $f$ is continuous, then $|\phi(T)|=\left|f\left(T x_{0}\right)\right| \leq c p_{\beta}\left(T x_{0}\right) \leq c q_{\beta}^{M}(T)$, where $M$ is an arbitrary bounded set in $X$ containing $x_{0}$. Let $\phi$ be $\tau_{b}$-continuous on $\mathscr{L}(X)$. Choose any $x \in X$. By the Hahn-Banach theorem there is some $g \in X^{\prime}$ such that $g\left(x_{0}\right)=1$ and let $S=x \otimes g$, hence $S x_{0}=x$. By continuity of $\phi$ there exist a seminorm $q_{\alpha}^{M}$ and $c>0$ such that $|f(x)|=\left|f\left(S x_{0}\right)\right|=|\phi(S)| \leq c q_{\alpha}^{M}(S)=c \sup _{y \in M} p_{\alpha}(g(y) x) \leq c_{1} p_{\alpha}(x) \sup _{y \in M} p_{\gamma}(y)=$ $d_{\gamma} p_{\alpha}(x)$ for some constant $d_{\gamma}>0$.

Proposition 2.17. Let $X$ be a locally convex space, $\mathscr{S}$ a semigroup in $\mathscr{L}(X)$, and $\phi$ a $\tau_{b}$ continuous coordinate functional on $\mathscr{L}(X)$. Then $\mathscr{S}$ is reducible if one of the following conditions holds:

(i) $\phi$ is constant on $\mathscr{Y}$,

(ii) $\phi$ is multiplicative on $\mathscr{Y}$.

The proof is the same as for the normed space (see [11, Lemma 8.2.8]).

Corollary 2.18. Let $X$ be a locally convex space, $\mathscr{E}$ a noncommutative family in $\mathscr{L}(X)$, and $\phi$ a $\tau_{b}$-continuous coordinate functional which is permutable on $\mathscr{E}$, then $\mathscr{E}$ is reducible.

Proof. Since permutability is inherited by passing to an algebra (see [11, page 28]), we can assume that $\mathscr{E}$ is an algebra. Let us choose $A, B$ in $\mathscr{E}$ such that $C:=A B-B A \neq 0$. Then it is easy to see that $\phi$ is equal to zero on ideal $\mathscr{F}$ generated by $C$ and by the previous proposition $\mathscr{F}$ is reducible, hence $\mathscr{E}$ is reducible.

Lemma 2.19. Let $X$ be a locally convex space and $A, B \in \mathscr{L}(X)$ such that $\sigma(A B)$ is bounded. Then $\sigma(A B) \backslash\{0\}=\sigma(B A) \backslash\{0\}$ and $r(A B)=r(B A)$.

Proof. It is easy to see that if for $\lambda \neq 0$ there exists $C:=(\lambda I-A B)^{-1} \in \mathscr{L}(X)$. Then we also have that $(\lambda I-B A)^{-1}=\lambda^{-1}(I+B C A) \in \mathscr{L}(X)$. Hence also $\sigma(B A)$ is bounded and both equalities follow.

A locally convex space is called $H$-locally convex if its topology can be defined by a calibration $P$ such that each $p_{\alpha} \in P$ is generated by a semiscalar product: $p_{\alpha}^{2}(x)=(x, x)_{\alpha}$ 
(see, e.g., [10]). In $H$-locally convex spaces the trace functional is well-defined on nuclear operators and a generalization to these spaces of Lidskii's theorem holds, which says that the trace of nuclear operator is equal to the sum of its eigenvalues, (see [7]). By this theorem and by the above lemma we also have $\operatorname{tr}(A B)=\operatorname{tr}(B A)$ for each pair of nuclear operators $A$ and $B$ acting on an $H$-locally convex space.

THEOREM 2.20. Let $\mathscr{S}$ be a family of nuclear operators on a barreled H-locally convex space. Then $\mathscr{S}$ is triangularizable if and only if the trace functional is permutable on $\mathscr{S}$.

Proof. We can verify as in [11, Lemma 2.1.14] that the permutability of the trace functional is equivalent to the condition $\operatorname{tr}(A B C)=\operatorname{tr}(A C B)$, for all $A, B, C \in \mathscr{Y}$. Let $\mathscr{S}$ be triangularizable. Then for the diagonal elements the following relations hold:

$$
d_{j}(A B C)=d_{j}(A) d_{j}(B) d_{j}(C)=d_{j}(A C B) ; \quad A, B, C \in \mathscr{S}
$$

(see [9]), where it is also shown that the nonzero eigenvalues coincide with nonzero diagonal elements for each operator of $\mathscr{Y}$. Thus, for any $A, B, C \in \mathscr{S}$ we have

$$
\operatorname{tr}(A B C)=\sum \lambda_{j}(A B C)=\sum d_{j}(A B C)=\sum d_{j}(A C B)=\operatorname{tr}(A C B) .
$$

Let the trace be permutable on $\mathscr{S}$. Then it is also permutable on the algebra $\mathscr{A}$ generated by $\mathscr{Y}$. Hence $\operatorname{tr}(A(B C-C B))=0, A, B, C \in \mathcal{A}$. Taking $A=(B C-C B)^{n-1}$, for $n \in \mathbb{N}$ we obtain $\operatorname{tr}\left((B C-C B)^{n}\right)=0, n \in \mathbb{N}$. Denote $T:=B C-C B$, since $T \in \mathscr{Y}(X)$, we have $\lambda_{j}\left(T^{n}\right)=\lambda_{j}(T)^{n}$ for each $j$ (see [12]). Hence, by Lidskii's theorem we have

$$
\sum_{j} \lambda_{j}(T)^{n}=\sum_{j} \lambda_{j}\left(T^{n}\right)=\operatorname{tr}\left(T^{n}\right)=0, \quad n \in \mathbb{N} .
$$

As in [4] it follows that $\lambda_{j}(T)=0$ for each $j$, thus, $\sigma(B C-C B)=\{0\}$ for each pair $B, C \in$ $\mathscr{A}$ and by [5, Theorem 2], $\mathscr{A}$ is triangularizable and the same holds for $\mathscr{S}$.

Corollary 2.21. Let $\mathscr{S}$ be a family of nuclear operators on a barreled H-locally convex space. Then $\mathscr{S}$ is triangularizable if the trace functional satisfies one of the following conditions:

(i) it is multiplicative on $\mathscr{T}$,

(ii) it is constant on $\mathscr{Y}$.

The following result is a generalization of the so-called "downsizing lemma" from [11].

LeMma 2.22. Let $X$ be a barreled locally convex space, $\mathscr{Y}$ a semigroup of compact operators on $X$ and $\mathscr{P}$ a property on $\mathscr{S}$ such that

(i) each subsemigroup in $\mathscr{S}$ has the property $\mathscr{P}$,

(ii) $\left.\mathscr{S}\right|_{X_{0}}$, has the property $\mathscr{P}$, where $X_{0}=\overline{\operatorname{span}\{\mathscr{R}(S), S \in \mathscr{S}\}}$,

(iii) $\overparen{\mathbb{R}^{+} \mathscr{S}}$ has the property $\mathscr{P}$.

Let $\mathscr{S}$ be irreducible. Then there exist a natural number $k \geq 2$ and an idempotent operator $E \in \mathscr{L}(X)$ of rank $k$, such that $\mathscr{S}$ contains a subsemigroup $\mathscr{Y}_{0}$ with properties: $\mathscr{Y}_{0}=E \mathscr{Y}_{0}$, $\left.\mathscr{S}_{0}\right|_{\mathscr{R}(E)}$ is irreducible in $\mathscr{L}\left(\mathbb{C}^{k}\right)$ and it has the property $\mathscr{P}$. Moreover, if $\min \{\operatorname{rank}(F): F \in$ $\left.\widetilde{\mathbb{R}^{+} \mathscr{S}}\right\}>1$, the operator $E$ can be chosen from $\widetilde{\mathbb{R}^{+} \mathscr{S}}$. 
Proof. By [6, Theorem 3], not all operators in $\mathscr{S}$ are quasinilpotent, hence by Proposition 2.4, $\widetilde{\mathbb{R}^{+\mathscr{S}}}$ contains a nonzero finite-rank operator. By [6, Proposition 2], $\hat{\mathscr{S}}:=\widetilde{\mathbb{R}^{+\mathscr{Y}}}$ is also a semigroup. Then the proof is the same as for the normed space (see [11, Lemma 8.2.13]), where we take $\hat{\mathscr{S}}$ instead of $\mathscr{Y}$.

Proposition 2.23. Let $X$ be a barreled locally convex space and $\mathscr{Y}$ a semigroup of compact operators on $X$. If $\mathscr{S}^{k}$ is triangularizable for some $k \in \mathbb{N}$, then $\mathscr{Y}$ is triangularizable.

Proof. If $\mathscr{S}^{k}=\{0\}$, then by [6, Theorem 3], $\mathscr{Y}$ is reducible. Let $\mathscr{S}^{k} \neq\{0\}$ and let $\mathscr{S}^{k}$ be triangularizable, then $\mathscr{S}$ is reducible since $\mathscr{S}^{k}$ is an ideal in $\mathscr{S}$. The triangularizability of a family of compact operators $\mathscr{S}^{k}$ is inherited by quotients which follows in the same manner as in the proof of [11, Theorem 7.3.9] for the normed space. Then applying the triangularization lemma [5] the triangularization of $\mathscr{S}$ follows.

Theorem 2.24. Let $X$ be a barreled locally convex space and $\mathscr{S}$ a semigroup of compact operators on $X$. If $A B-B A$ is quasinilpotent for every $A, B \in \mathscr{Y}$, then $\mathscr{T}$ is triangularizable.

Proof. By [5, Lemma 5], the quasinilpotency is inherited by quotients for compact operators. So, by triangularizing lemma it suffices to prove the reducibility of the semigroup $\mathscr{S}$. Let us verify the conditions of Lemma 2.22. The condition (i) is obvious. Denoting by $X_{0}$ the closed span of $\{\mathscr{R}(S): S \in \mathscr{S}\}$, then for $A, B \in \mathscr{Y}$ it easy to see that $\left.\left.A\right|_{X_{0}} B\right|_{X_{0}}-\left.\left.B\right|_{X_{0}} A\right|_{X_{0}}=\left.(A B-B A)\right|_{X_{0}}$ and it is clear that $\left.(A B-B A)\right|_{X_{0}}$ is also quasinilpotent, hence (ii) holds. By [5, Theorem 1], quasinilpotency is inherited by $\widetilde{\mathbb{R}^{+} \mathscr{S}}$ and so (iii) holds. If $\mathscr{S}$ were irreducible, then by Lemma 2.22 , there would exist a subsemigroup $\mathscr{S}_{0}$ of $\mathscr{Y}$ such that $\mathscr{Y}_{0} \mid \mathscr{R}(E)$ would be irreducible in $\mathscr{L}\left(\mathbb{C}^{k}\right)$, which is impossible $[11$, Theorem 4.4.12].

Let $\mathscr{E}$ be a semigroup in $\mathscr{L} B(X)$. It is known (see, e.g., [12]) that the spectrum for each $T \in \mathscr{L} B(X)$ is bounded. We say that the spectrum is submultiplicative on $\mathscr{E}$ if $\sigma(A B) \subset$ $\sigma(A) \sigma(B)=\{\lambda \mu: \lambda \in \sigma(A), \mu \in \sigma(B)\}$ for all $A, B \in \mathscr{E}$.

Theorem 2.25. Let $X$ be an infinite-dimensional barreled locally convex space and $\mathscr{S}$ a semigroup of compact operators on $X$ with the property that the spectrum is submultiplicative on $\mathscr{Y}$. Then $\mathscr{Y}$ is reducible.

Proof. Let us denote by $\hat{\mathscr{S}}:=\widetilde{\mathbb{R}^{+} \mathscr{Y}}$. Suppose that $\mathscr{Y}$ is irreducible, then $\hat{\mathscr{Y}}$ is irreducible too. By [6, Proposition 3] there exists nonzero finite-rank idempotent operator $E \in \hat{\mathscr{S}}$ which has minimal rank $m$. Denoting $\mathscr{S}_{0}=\hat{\mathscr{S}} E \hat{\mathscr{S}}$, this is an ideal in $\hat{\mathscr{S}}$ and all operators in $\mathscr{Y}_{0}$ have a rank equal to $m$ or 0 . Thus, $\mathscr{S}_{0}$ is irreducible and then the rest of the proof is the same as for the normed space (see [11, Theorem 8.3.5]), where we take $\mathscr{Y}_{0}$ instead of $\mathscr{Y}$.

Theorem 2.26. Let $X$ be an infinite-dimensional barreled locally convex space and $\mathscr{S}$ a semigroup of compact operators with the following property: $\sigma(S) \subset\{0,1\}$ for every $S \in \mathscr{S}$ and if $1 \in \sigma(S T)$, for $S, T \in \mathscr{Y}$, let $1 \in \sigma(S) \cap \sigma(T)$. Then $\mathscr{S}$ is reducible. 
Proof. We can assume that $X$ is not normable (see [11, Theorem 8.3.8]). Since each $A \in \mathscr{S}$ is locally bounded, it has 0 in his spectrum [12]. With the above assumption the submultiplicativity of spectrum on $\mathscr{Y}$ follows, so $\mathscr{Y}$ is reducible by the previous theorem.

Theorem 2.27. Let $X$ be a barreled locally convex space and $\mathscr{S}$ a semigroup of compact idempotent operators. Then $\mathscr{S}$ is triangularizable.

Proof. In view of [11, Theorem 2.3.5] let $X$ be infinite-dimensional. A quotient of an idempotent is, clearly, idempotent operator and a quotient of compact operator is again compact (see [5]). So, by the triangularization lemma, it suffices to prove the reducibility. Clearly, for an idempotent $S$ and $\lambda \neq 0, \neq 1$, there exists $(\lambda I-S)^{-1}=I / \lambda-S /(\lambda(1-\lambda)) \in$ $\mathscr{L}(X)$, hence $\sigma(S) \subset\{0,1\}$. If $1 \in \sigma(S T)$ for $S, T \in \mathscr{Y}$, then $S$ and $T$ are nonzero idempotent, hence $1 \in \sigma(S) \cap \sigma(T)$. Thus, the conditions of the preceding theorem are fulfilled and the reducibility of $\mathscr{S}$ follows.

For a semiball $U_{\gamma}, \gamma \in \Delta$, let us denote by $\mathscr{L}_{\gamma}(X)$ the family of all continuous linear operators $T$ on $X$, for which $T\left(U_{\gamma}\right)$ is a bounded set. Clearly, this is a subspace and left ideal in $\mathscr{L}(X)$. For each $T \in \mathscr{L}_{\gamma}(X)$ we have $T\left(U_{\gamma}\right) \subset \lambda_{\gamma} U_{\gamma}$ for some $\lambda_{\gamma}>0$, hence $T\left(J_{\gamma}\right) \subset$ $J_{\gamma}$, thus, operator $T_{\gamma}$ is well defined on $X_{\gamma}$. For some fixed $T \in \mathscr{L}_{\gamma}(X)$ the convergence of some sequence in $\mathscr{L}(X)$ is inherited to the operator sequence on the quotient space $X_{\gamma}$ in the following sense.

Lemma 2.28. Let $X$ be a locally convex space, $T \in \mathscr{L}_{\gamma}(X)$ for some $\gamma \in \Delta$ and $\left\{S_{n}\right\} \subset \mathscr{L}(X)$ a sequence which is $\tau_{b}$-convergent to some $S$ in $\mathscr{L}(X)$. Then $\left\{\left(S_{n} T\right)_{\gamma}\right\}$ is convergent to $(S T)_{\gamma}$ with respect to the norm $\|\cdot\|_{\gamma}$ in $X_{\gamma}$.

Proof. Since $M:=T\left(U_{\gamma}\right)$ is a bounded set, we have $\left\|\left(S_{n} T\right)_{\gamma}-(S T)_{\gamma}\right\|_{\gamma}=\sup _{x \in U_{\gamma}} p_{\gamma}\left(\left(S_{n} T\right.\right.$ $-S T) x)=\sup _{y \in M} p_{\gamma}\left(\left(S_{n}-S\right) y\right)=q_{\gamma}^{M}\left(S_{n}-S\right) \rightarrow 0$, as $n \rightarrow \infty$.

It is well known that in a normed space the spectral radius is continuous on the set of compact operators (see, e.g., [11]), but this is not the case for general locally convex spaces. Let us take as an example $X=s$, the space of all real sequences $\left\{x_{n}\right\}$ with the topology generated by seminorms $P=\left\{p_{m}: m \in \mathbb{N}\right\}$, where $p_{m}(x)=\sup \left\{\left|x_{j}\right|: j \leq m\right\}$, $x \in X$ and a sequence of operators $\left\{T_{n}\right\}$ defined by $T_{n}\left(x_{1}, x_{2}, \ldots\right)=\left(0,0, \ldots, x_{n}, 0,0, \ldots\right)$. It is easy to see that all $T_{n}$ are compact and $T_{n} \stackrel{\tau_{b}}{\rightarrow} T$, where $T=0$. Hence, $r\left(T_{n}\right)=1$ for all $n \in \mathbb{N}$, but $r(T)=0$. We will prove the continuity of spectral radius in a special case.

Lemma 2.29. Let $X$ be a locally convex space, $T \in \mathscr{K}(X)$ and $\left\{S_{n}\right\}$ a sequence of continuous operators which is $\tau_{b}$-convergent to $S \in \mathscr{L}(X)$. Then

$$
r\left(S_{n} T\right) \longrightarrow r(S T), \quad r\left(T S_{n}\right) \longrightarrow r(T S), \quad \text { as } n \longrightarrow \infty
$$

Proof. Since $T$ is locally bounded, there is some $\gamma \in \Delta$ such that $T \in \mathscr{L}_{\gamma}(X)$ and also $A T \in \mathscr{L}_{\gamma}(X)$ for each $A \in \mathscr{L}(X)$. The corresponding operator $T_{\gamma}$ is also compact on $X_{\gamma}$ and for the spectral radius we have $r(T)=r\left(T_{\gamma}\right)$ (see [2]). Then, by Lemma 2.28 and by continuity of the spectral radius for a sequence of compact operators acting on a normed 
space, we obtain $r\left(S_{n} T\right)=r\left(\left(S_{n} T\right)_{\gamma}\right) \rightarrow r\left((S T)_{\gamma}\right)=r(S T)$, as $n \rightarrow \infty$. By Lemma 2.19 we have also $r\left(T S_{n}\right) \rightarrow r(T S)$, as $n \rightarrow \infty$.

Lemma 2.30. Let $X$ be a locally convex space, $\mathscr{S}$ a semigroup in $\mathscr{L}(X)$ and $A, B$ some nonzero members in $\mathscr{S}$ such that $B \mathscr{Y} A=\{0\}$. Then $\mathscr{Y}$ is reducible.

Proof. If $\mathscr{Y} \mathscr{R}(A)=\{0\}$, then it is easy to see that $\overline{\mathscr{R}(A)}$ is a nontrivial invariant subspace for $\mathscr{Y}$. If $\mathscr{Y} \mathscr{R}(A) \neq\{0\}$, then the closed span of this set is a nontrivial invariant subspace for $\mathscr{S}$.

Lemma 2.31. Let $X$ be a locally convex space and $\mathscr{Y}$ an irreducible semigroup of compact operators on $X$. If spectral radius is submultiplicative on $\mathscr{S}$ then no nonzero product $\widetilde{S} \widetilde{T}$, where $\tilde{S}, \widetilde{T} \in \tilde{\mathscr{T}}$, is quasinilpotent.

Proof. Let us denote $\mathscr{\mathscr { S }}=\{\tilde{S} \widetilde{T}: \widetilde{S}, \widetilde{T} \in \tilde{\mathscr{S}}, r(\tilde{S} \widetilde{T})=0\}$. This is an outer ideal of $\mathscr{S}$. Indeed, choose any product $\tilde{S} \widetilde{T} \in \mathscr{F}$ and $C \in \mathscr{G}$, then there exist two sequences $\left\{S_{n}\right\}$ and $\left\{T_{m}\right\}$ in $\mathscr{S}$ such that $S_{n} \stackrel{\tau_{b}}{\rightarrow} \widetilde{S}$ and $T_{m} \stackrel{\tau_{b}}{\rightarrow} \widetilde{T}$. For each pair of operators $S_{n}, T_{m}$ we have

$$
r\left(C S_{n} T_{m}\right) \leq r(C) r\left(S_{n} T_{m}\right) ; \quad n, m \in \mathbb{N} .
$$

Using Lemma 2.29 twice we obtain $r(C \tilde{S} \tilde{T}) \leq r(C) r(\tilde{S} \tilde{T})=0$, hence, $C \tilde{S} \tilde{T} \in \mathscr{g}$. Since $r(\tilde{S} \tilde{T} C)=r(C \tilde{S} \tilde{T})$, also $\tilde{S} \tilde{T} C \in \mathscr{F}$. If $\mathscr{F} \neq\{0\}$, by [6, Theorem 3], it would be reducible and then by [6, Lemma 5], $\mathscr{S}$ would be reducible too. Thus, $\mathscr{E}=\{0\}$.

THEOREM 2.32. Let $\mathscr{S}$ be an irreducible semigroup of compact operators on a barreled locally convex space. If spectral radius is submultiplicative on $\mathscr{Y}$, then it is multiplicative on $\mathscr{S}$.

Proof. Since the spectral radius is homogenous for the nonnegative scalars, one can suppose $\mathbb{R}^{+} \mathscr{S}=\mathscr{Y}$. By [6], $\tilde{\mathscr{S}}$ is again a semigroup. Let us prove that it has no quasinilpotent element. Suppose, to the contrary, that there is a nonzero quasinilpotent operator $\tilde{T}$ in $\tilde{\mathscr{S}}$. Then also $r\left(\widetilde{T}^{2}\right)=0$, hence by the previous lemma, $\widetilde{T}^{2}=0$. Consequently $r(\tilde{T} \tilde{S} \tilde{T})=r\left(\tilde{S} \tilde{T}^{2}\right)=0$ for any $\tilde{S} \in \tilde{\mathscr{S}}$. Thus $\tilde{T} \tilde{\mathscr{T}} \widetilde{T}=\{0\}$, hence, by Lemma $2.30, \tilde{\mathscr{S}}$ is reducible, which is a contradiction. Choose any $A, B \in \mathscr{Y}$, where we can assume $r(A)=$ $r(B)=1$. By Proposition 2.4 there exist two nonzero finite-rank idempotents $E, F \in \tilde{\mathscr{S}}$ such that $A^{n_{i}} \stackrel{\tau_{b}}{\rightarrow} E$ and $B^{m_{k}} \stackrel{\tau_{b}}{\rightarrow} F$ for some sequences of integers $\left\{n_{i}\right\}$ and $\left\{m_{k}\right\}$. Let us prove that $E F \neq 0$. If $E F=0$, then $r(F \tilde{S} E)=r(\widetilde{S} E F)=0$ and by the above lemma it would be $F \tilde{S} E=0$ for each $\tilde{S} \in \tilde{\mathscr{T}}$, hence $F \tilde{\mathscr{S}} E=\{0\}$ and by Lemma $2.30, \tilde{\mathscr{S}}$ would be reducible. In the sequel, let us prove the following inequality:

$$
r\left(E F^{2} E\right) \leq r(E F)^{2}
$$

For each pair of operators from the sequences defining $E$ and $F$ we have by assumption $r\left(A^{n_{i}} B^{2 m_{k}} A^{n_{i}}\right) \leq r\left(A^{n_{i}} B^{m_{k}}\right) r\left(B^{m_{k}} A^{n_{i}}\right)$ and by Lemma 2.29 we obtain the above inequality. Similarly, we have $r\left(A^{n_{i}} B^{m_{k}}\right)=r\left(A B B^{m_{k}-1} A^{n_{i}-1}\right) \leq r(A B) r(A)^{n_{i}-1} r(B)^{m_{k}-1}=r(A B)$ and by Lemma 2.29 we obtain

$$
r(E F) \leq r(A B) .
$$


From the idempotency of $E$ and $F$ and by the inequality (2.10) it follows that:

$$
r(E F)=r\left(E^{2} F^{2}\right)=r\left(E F^{2} E\right) \leq r(E F)^{2} .
$$

Thus, we have $1 \leq r(E F) \leq r(A B) \leq r(A) r(B)=1$, and the multiplicativity of the spectral radius follows.

Theorem 2.33. Let $X$ be a barreled locally convex space, $\mathscr{Y}$ a semigroup of compact operators on $X$ such that each $S \in \mathscr{Y}$ is a nonnegative scalar multiple of an idempotent operator and let spectral radius be submultiplicative on $\mathscr{S}$. Then $\mathscr{S}$ is triangularizable.

Proof. Let us prove that $\mathscr{S}$ is reducible. Denote $\mathscr{Y}_{0}=\{S / r(S): S \in \mathscr{T}, S \neq 0\} \cup\{0\}$. Clearly, $\mathscr{S}_{0}$ is reducible if and only if $\mathscr{S}$ is reducible. Suppose that $\mathscr{S}$ is irreducible. Then, by Theorem 2.32 the spectral radius is multiplicative on $\mathscr{S}$. Consequently, $\mathscr{Y}_{0}$ is a semigroup of compact idempotents. By Theorem $2.27, \mathscr{S}_{0}$ is reducible. Thus, $\mathscr{S}$ is reducible and by triangularization lemma it is triangularizable.

In view of Lemma 2.19 it is easy to see that the spectral radius is permutable on a semigroup $\mathscr{S}$ if and only if $r(A B C)=r(A C B)$ for all $A, B \in \mathscr{Y}$.

THeorem 2.34. Let $X$ be a locally convex space and $\mathscr{S}$ a semigroup of compact operators on $X$. Then spectral radius is submultiplicative on $\mathscr{S}$ if and only if it is permutable on $\mathscr{S}$.

Proof. We will use the property $r(T)=r\left(T_{\gamma}\right)$ for $T \in \mathscr{L}_{\gamma}(X), \gamma \in \Delta$ (see [2]). With no loss of generality we may assume that the calibration $P \in \mathscr{P}(X)$ is directed, that is for each $p_{\alpha}, p_{\beta} \in P$ there is some $p_{\gamma} \in P$ such that $p_{\alpha} \leq p_{\gamma}$ and $p_{\beta} \leq p_{\gamma}$. Let $r$ be permutable on $\mathscr{S}$. Choose any $A, B \in \mathscr{Y}$. Since they are locally bounded and $P$ is directed, there exists $p_{\gamma} \in P$ such that $A, B \in \mathscr{L}_{\gamma}(X)$. Denote by $\mathscr{Y}_{0}^{\gamma}$ the semigroup generated by $A_{\gamma}, B_{\gamma}$. By [6, Lemma 1] spectral radius is also permutable on $\mathscr{S}_{0}^{\gamma}$ and by [11, Theorem 8.6.3] it is submultiplicative and then $r(A B)=r\left(A_{\gamma} B_{\gamma}\right) \leq r\left(A_{\gamma}\right) r\left(B_{\gamma}\right)=r(A) r(B)$. Let $r$ be submultiplicative on $\mathscr{S}$. For any $A, B, C \in \mathscr{Y}$ there is some $p_{\gamma} \in P$ such that $A, B, C \in \mathscr{L}_{\gamma}(X)$, then on the semigroup $\mathscr{S}_{1}^{\gamma}$ generated by $A_{\gamma}, B_{\gamma}$, and $C_{\gamma}$ the submultiplicativity implies the permutability of $r$ and similarly as above we obtain $r(A B C)=r(A C B)$.

Question. What are the conditions on a family of compact operators on a locally convex space yielding the continuity of the spectral radius on this family?

\section{References}

[1] K. Floret and J. Wloka, Einführung in die Theorie der lokalkonvexen Räume, Lecture Notes in Mathematics, no. 56, Springer, Berlin, 1968.

[2] F. Garibay Bonales and R. Vera Mendoza, Extending the formula to calculate the spectral radius of an operator, Proc. Amer. Math. Soc. 126 (1998), no. 1, 97-103.

[3] D. Kalnins, Sous-espaces hyperinvariant d'un operateur compact, C. R. Acad. Sci. Paris Sér. A 288 (1979), 115-116.

[4] A. Katavolos and H. Radjavi, Simultaneous triangularization of operators on a Banach space, J. London Math. Soc. (2) 41 (1990), no. 3, 547-554.

[5] E. Kramar, Triangularization of some families of operators on locally convex spaces, Comment. Math. Univ. Carolin. 42 (2001), no. 3, 499-506. 
[6] - On reducibility of Volterra semigroups on locally convex spaces, Acta Sci. Math. (Szeged) 70 (2004), no. 1-2, 289-298.

[7] G. L. Litvinov, On the traces of linear operators in locally convex spaces, Selecta Math. Soviet. 8 (1989), no. 3, 203-212.

[8] G. L. Litvinov and V. I. Lomonosov, Density theorems in locally convex spaces and their applications, Trudy Sem. Vektor. Tenzor. Anal. (1981), no. 20, 210-227 (Russian).

[9] T.-W. Ma, On rank one commutators and triangular representations, Canad. Math. Bull. 29 (1986), no. 3, 268-273.

[10] T. Precupanu, Espaces linéaires à semi-normes hilbertiennes, An. Şti. Univ. “Al. I. Cuza” Iaşi Secț. I a Mat. (N.S.) 15 (1969), 83-93.

[11] H. Radjavi and P. Rosenthal, Simultaneous Triangularization, Universitext, Springer, New York, 2000.

[12] P. Uss, Sur les opérateurs bornés dans les espaces localement convexes, Studia Math. 37 (1970/1971), 139-158.

Edvard Kramar: Faculty of Mathematics and Physics, University of Ljubljana, Jadranska 19, 1000 Ljubljana, Slovenia

E-mail address: edvard.kramar@fmf.uni-lj.si 


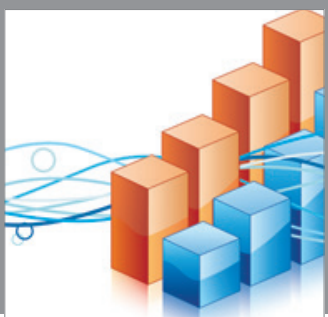

Advances in

Operations Research

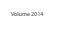

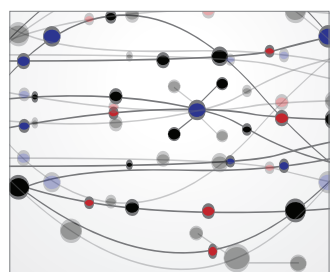

\section{The Scientific} World Journal
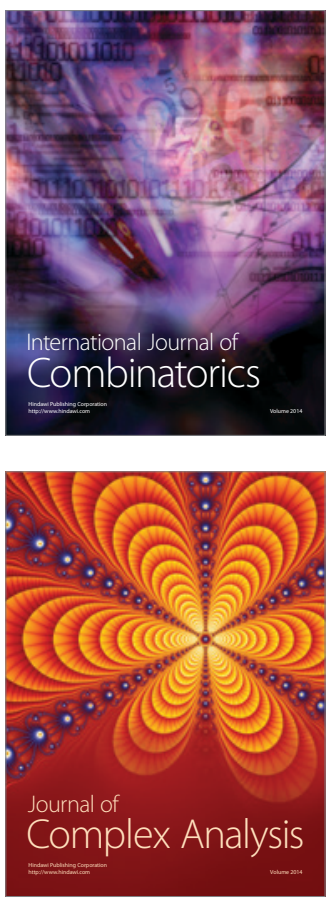

International Journal of

Mathematics and

Mathematical

Sciences
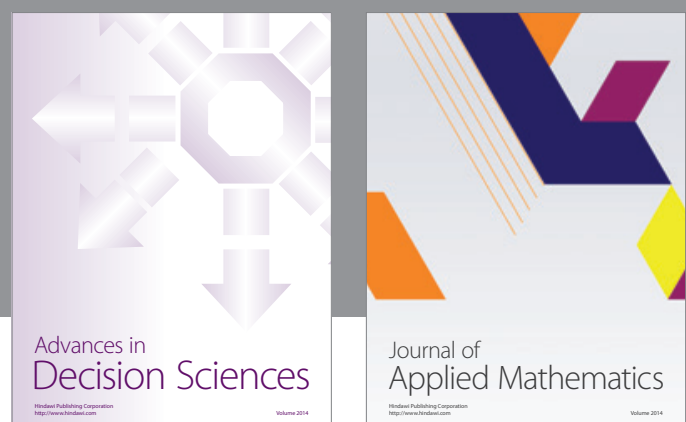

Journal of

Applied Mathematics
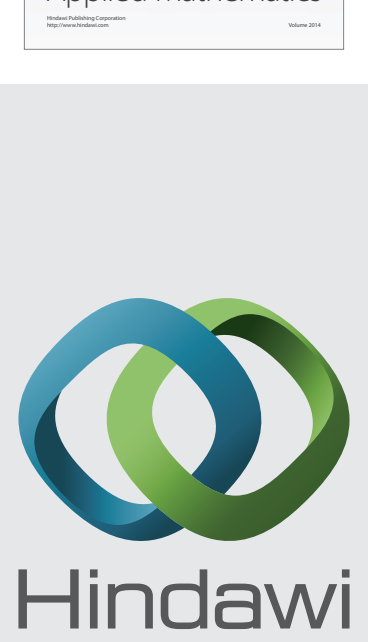

Submit your manuscripts at http://www.hindawi.com
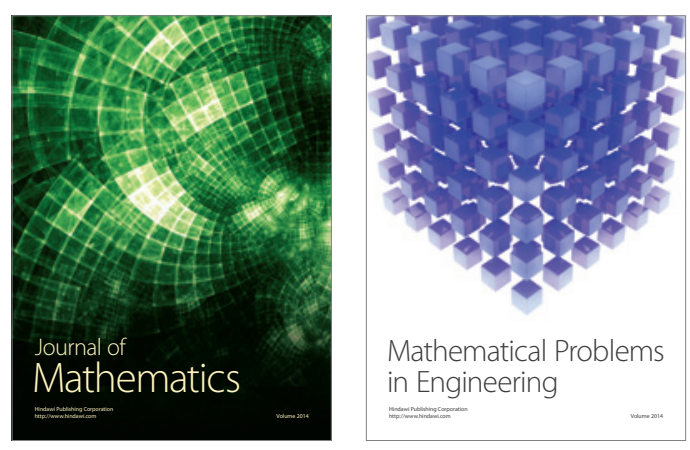

Mathematical Problems in Engineering
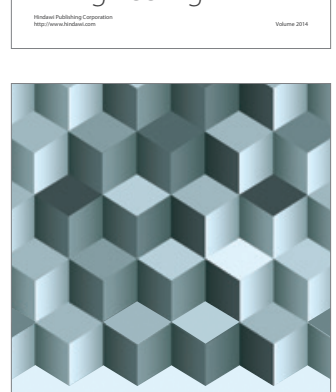

Journal of

Function Spaces
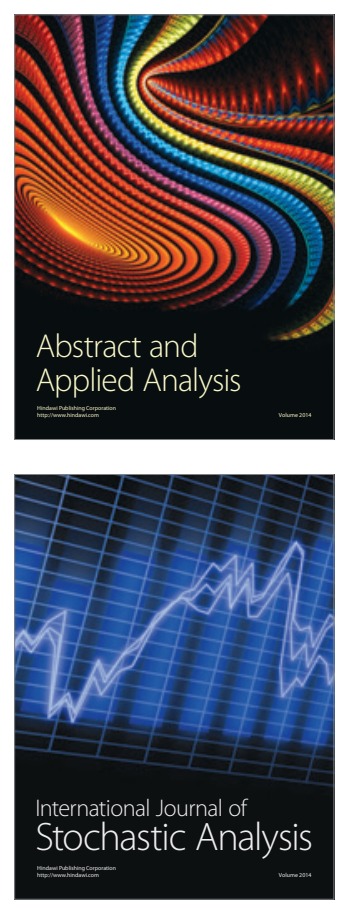

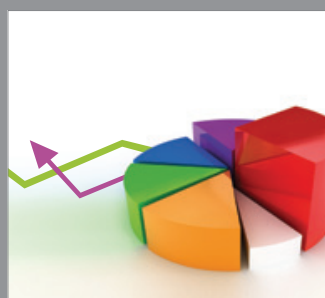

ournal of

Probability and Statistics

Promensencen
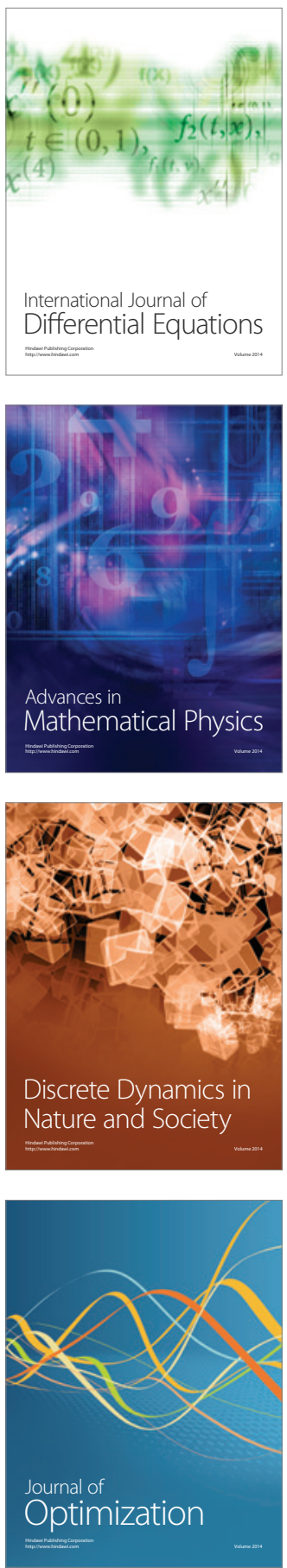